\title{
Article \\ Enhancing the Triboelectric Nanogenerator Output by Micro Plasma Generation in a Micro-Cracked Surface Structure
}

\author{
Jinhyoung Park ${ }^{1}\left(\mathbb{D}\right.$, Hanchul Cho ${ }^{2, *} \mathbb{C}$ and Yong-Seok Lee ${ }^{3, *}$ \\ 1 School of Mechatronics Engineering, Korea University of Technology \& Education, 600, Chungjeol-ro, \\ Byeongcheon-myeon, Dongnam-gu, Cheonan-si 31253, Korea; jhpark98@koreatech.ac.kr \\ 2 Precision Mechanical Process and Control R\&D Group, Korea Institute of Industrial Technology (KITECH), \\ 42-7, Baegyang-daero 804 beon-gil, Sasang-gu, Busan 46938, Korea \\ 3 Department of Mechanical Engineering, School of Industrial and Mechanical Engineering, \\ The University of Suwon, 17, Wauan-gil, Bongdam-eup, Hwaseong-si 18323, Korea \\ * Correspondence: hc.cho@kitech.re.kr (H.C.); yongseoklee@suwon.ac.kr (Y.-S.L.)
}

check for

updates

Citation: Park, J.; Cho, H.; Lee, Y.-S. Enhancing the Triboelectric Nanogenerator Output by Micro Plasma Generation in a Micro-Cracked Surface Structure. Appl. Sci. 2021, 11, 4262. https:// doi.org/10.3390/app11094262

Received: 8 April 2021

Accepted: 3 May 2021

Published: 8 May 2021

Publisher's Note: MDPI stays neutral with regard to jurisdictional claims in published maps and institutional affiliations.

Copyright: (c) 2021 by the authors. Licensee MDPI, Basel, Switzerland. This article is an open access article distributed under the terms and conditions of the Creative Commons Attribution (CC BY) license (https:// creativecommons.org/licenses/by/ $4.0 /)$.

\begin{abstract}
Energy harvesting, especially for powering low-power internet-of-things (IoT) devices, is gaining attention in recent years. Triboelectric nanogenerators have been studied to improve the output by applying a structure that can concentrate electrons on the surface of the generator materials. For enhancing the triboelectrification output, we herein focused on the power output line. A method for increasing the amount of electrons on the power lead by potential difference and their acceleration was studied. A rod was shaken by external vibrations; the accumulated charges were discharged in a manner similar to that of a lightning rod. Micro plasma was generated when the rod made contact with the mating micro-cracked surface innumerable times. The micro-cracked surface was fabricated with a diamond tip moving horizontally to the surface. As the resistance of the micro plasma was close to zero, the amount of electron movement was instantaneously accelerated. This type of triboelectric generator can be fabricated in the form of an electric box. By using this triboelectric power amplifier, voltage can be amplified 2 to 3 times, and the current can be amplified 10 to 15 times; thus, enhanced energy harvesting efficiency is attained.
\end{abstract}

Keywords: energy harvesting; triboelectric nanogenerators; micro plasma

\section{Introduction}

Energy harvesting is a method of collecting and converting peripheral energy (i.e., energy which is likely to be neglected, such as gravitational energy, light energy, potential energy, electromagnetic wave energy, and vibration energy) into electricity. This method has been recently highlighted as a new solution to power low-power internet-of-things (IoT) devices [1,2]. The triboelectric generator (TENG) is an energy harvesting device with a simple structure and a high energy conversion efficiency. The triboelectric effect is used to harvest vibration energy. Since their introduction in 2012, TENGs have been studied by many researchers [3-5]. Triboelectric charging occurs when two different materials come in contact; consequently, innumerable materials in nature can be used. Accordingly, unlike the existing energy harvesting techniques that use hard materials to fabricate rotor type or piezoelectric generators, various flexible polymer materials can be utilized to fabricate a TENG.

Basically, a TENG generates electric charge flowing in a circuit via friction of materials and utilizes the electricity produced. For this reason, at least two materials are needed for triboelectrification. The electric energy generated by triboelectrification is proportional to the potential difference between the two materials and the quantity of electrons flowing in a circuit. A number of researchers have tried to increase the potential difference between materials, aiming to improve the output of triboelectrification [6-13]; material selection, surface charge, and the dielectric constant are the parameters that can be targeted for this 
purpose. The quantity of electrons can be increased by utilizing surface roughness, contact force/speed, and contact area [14-20]. These methods of improving the potential difference or the quantity of electrons are interrelated [21]. The electric current is the quantity of electrons flowing per unit of time and is transferred to a circuit through an electrode. Accordingly, if the resistance of the electrode is reduced, the feasibility of electron flow, that is, the current, will increase.

For enhancing the output of triboelectrification, this study proposed a method for modifying the electrode output, and not the energy harvester itself. The electrode output was modified by fabricating a simple sender that is applicable to most TENGs. The proposed method for improving the output focused on the following two points: first, the electric current is inversely proportional to the resistance of the electrode, and second, the electrons can be concentrated momentarily and transferred in a manner similar to the functioning of a lightning rod or an electron gun.

The first method of improving triboelectrification output utilizes micro plasma shapes. Generally, electrons accumulated by triboelectrification move to an electric circuit through a lead connected to an electrode. However, in this study, an air gap was introduced in the middle of the lead. This air gap was designed to continuously change its width under the influence of external vibrations. Electrons accumulated by triboelectrification moved to an electric circuit in the micro plasma state through the air gap; a dielectric breakdown may occur depending on the width of this air gap. As the micro plasma has a resistance close to zero, innumerable electrons can move instantaneously. In this manner, the output current of a TENG can increase.

\section{Materials and Methods}

Static electricity due to friction is more readily observed in a dry environment, increasing the feasibility of micro plasma generation. This study used the following structure to intentionally generate micro plasma by external vibration. A long rod-shaped metal structure and a mass for resonant motion by external vibration were placed at one end (Figure $1 \mathrm{a}-\mathrm{c}$ ). The metal rod was fabricated from a shape memory alloy ensuring high elasticity and restoring force. The mass was fabricated using light polyethylene terephthalate (PET). The other end was a ring-shaped contact surface structure. The micro-crack contact surface was fabricated using a diamond tip moving horizontally to the surface. The resulting needle-shaped surface facilitated the concentration of electrons. Both the mass and the metal rod were designed as a resonant structure to generate as much motion as possible by a single application of external force. The resonant frequency can be calculated using the following equation:

$$
\mathrm{f}(\mathrm{Hz})=\frac{1}{2 \pi} \sqrt{\frac{k}{m}}=\frac{1}{2 \pi} \sqrt{\frac{3 E I}{m l^{3}}} .
$$

Here, $\mathrm{f}(\mathrm{Hz})$ is the resonant frequency, $k$ is the spring constant, and $m$ is the weight of a mass. $E$ denotes the Young's modulus of bar, $l$ is the length of bar, and $I$ is the moment of inertia. In this design, the mass was $0.243 \mathrm{~g}$ in weight $(m=0.243 \mathrm{~g})$, and the rod was $50 \mathrm{~mm}$ in length $(l=50 \mathrm{~mm})$ and $0.3 \mathrm{~mm}$ in diameter $(\mathrm{d}=0.3 \mathrm{~mm})$.

In the design of this study, the resonant frequency was set to $7.05 \mathrm{~Hz}$, which belongs to the typical range of $1-10 \mathrm{~Hz}$ in nature. As a multiple number of contacts could occur in a single vibration, triboelectric charges could be effectively extracted from TENG to an electric circuit (Figure 1d). Charges accumulated in TENG were discharged as soon as the metal column and the internal surface of the ring were sufficiently close for the plasma discharge to occur. This occurred several times during a single external vibration event. The rough internal surface of the ring concentrated and easily emitted electrons, similar to a lightning rod or electron gun (Figure 1c). 
(a)

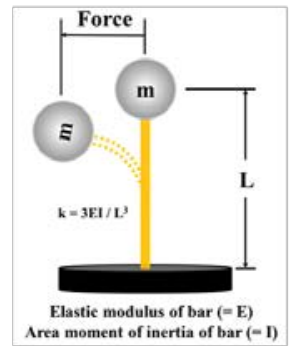

(d)

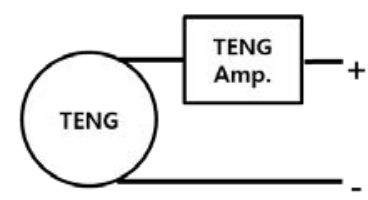

(b)

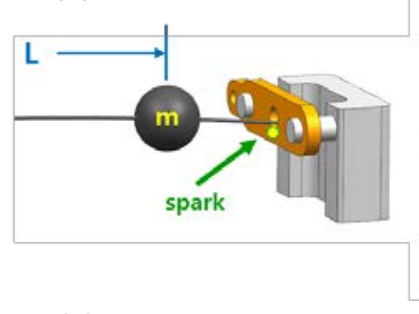

(c)

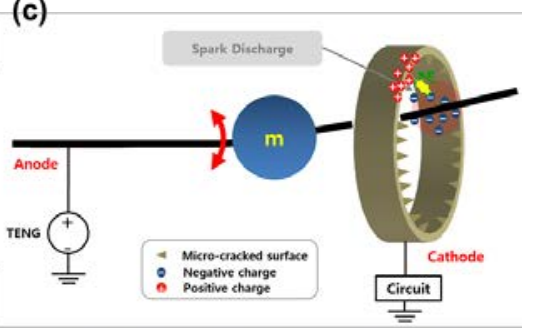

(e)

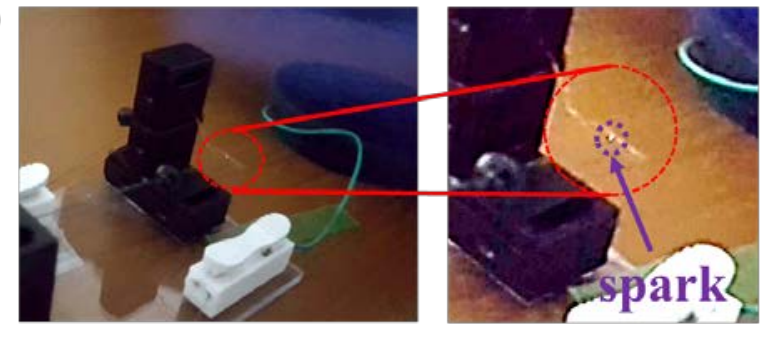

Figure 1. Basic principle of TENG output amplifier using micro plasma. (a) Basic vibration structure of mass and bar. (b) Design of vibration structure. (c) Working mechanism by which the charge is transferred during the process. (d) Schematic of circuit connection of TENG output amplifier. (e) Micro plasma occurring in the proposed TENG output amplifier.

Figure 1e shows the micro plasma occurring in the structure designed in this study. If a TENG has sufficient basic power, such micro plasma can occur several times owing to a single external force. Micro plasma has different intensities depending on whether the internal surface of the ring is smooth or rough. The TENG output amplifier (TENG Amp.) was designed by applying this theory, as illustrated in Figure 2a,c. Two terminals for connecting electrodes and a casing for protecting the interior part were added to the above basic structure. Figure $2 b, d-g$ show the shapes fabricated herein. The surface of TENG Amp. was observed to analyze the roughness by an optical microscope (VHX-7000, KEYENCE Corporation (Osaka, Japan)). The TENG output amplifier had dimensions of $30 \mathrm{~cm} \times 10 \mathrm{~cm} \times 5 \mathrm{~cm}$. One of the two terminals was connected to the output terminal of the TENG, and the other terminal was connected to the electric circuit. The effects of the TENG output amplifier are explained along with the test results in the next section.

Two types of TENG specimens were prepared for the test. The one specimen simply comprised a PDMS layer and an electrode. As shown in Figure 3a, the simple TENG specimen with the PDMS layer was fabricated by molding the PDMS layer with dimensions of $6 \mathrm{~cm} \times 6 \mathrm{~cm} \times 0.5 \mathrm{~cm}$ and placing it on a metal electrode. The other specimen was fabricated using a conductive sponge with a carbon-based porous PDMS structure. In this specimen, the CNT-PDMS conductive sponge was used as an electrode structure. Silicone rubber was adopted for negative charges. The contact/separation triboelectrification mode was applied. An Al plate connected to a pushing tester was used as a positively charged counterpart.

The conductive sponge was fabricated using a process illustrated in Figure $3 \mathrm{~b}$. Coarsegrained sugar was used to fabricate a porous structure. Porous CNT-PDMS nanocomposites were formed via the templating technique, which is cheap, simple, and environmentally friendly, using the following sequence: create sugar cube templates on Petri dishes depending on the weight percent of CNTs $14 \mathrm{wt} \%$, dry the sugar cube templates at $70{ }^{\circ} \mathrm{C}$ for $1 \mathrm{~h}$ in the oven, immerse the sugar cube templates on the PDMS solution prepared by mixing a base and a curing agent at a weight ratio of 10:1 for $1 \mathrm{~h}$ in vacuum, cure the PDMS solution at $70{ }^{\circ} \mathrm{C}$ for $1.5 \mathrm{~h}$, remove the sugar by immersing the samples in water for $2 \mathrm{~h}$, dry the samples overnight, apply a 0.3-mm-thick silicone rubber on the surface, and finally dry it for $1 \mathrm{~h}$. A copper wire with silver paste was employed as the electrode. 
(a)
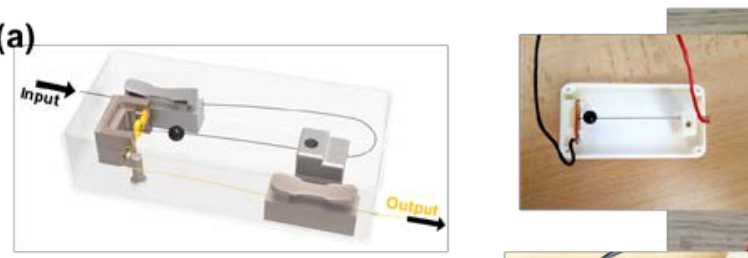

(b)

(c)
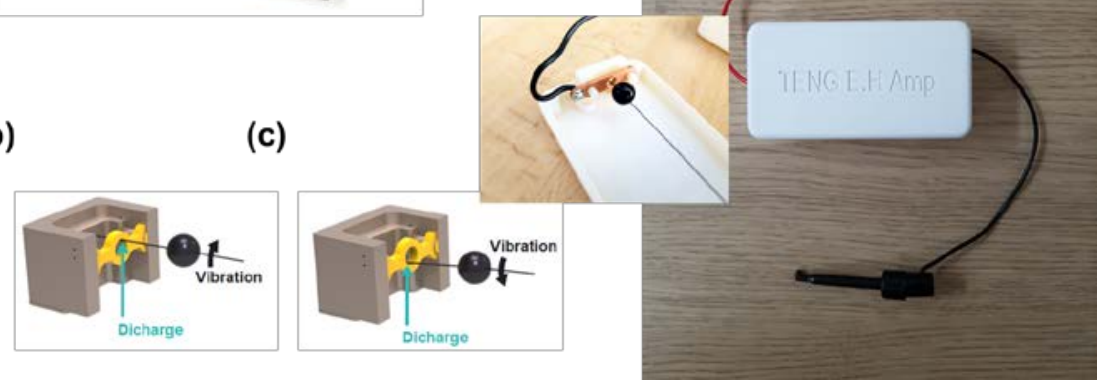

(d)

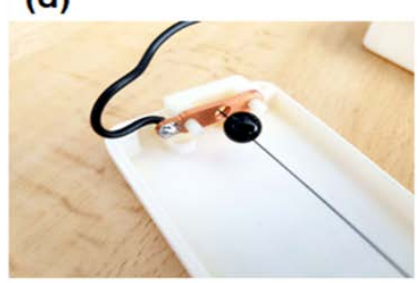

(f)

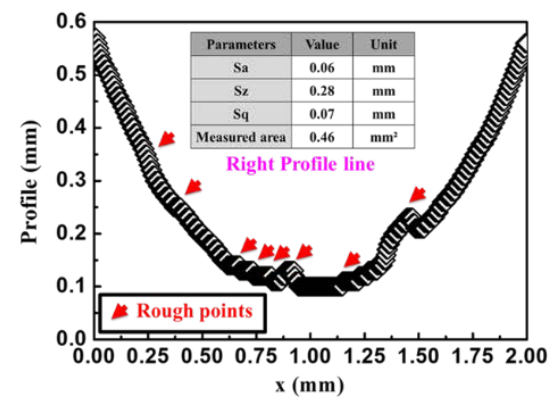

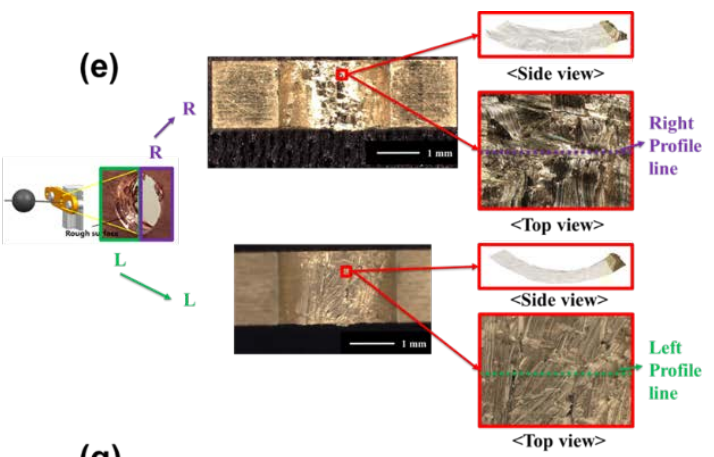

(g)

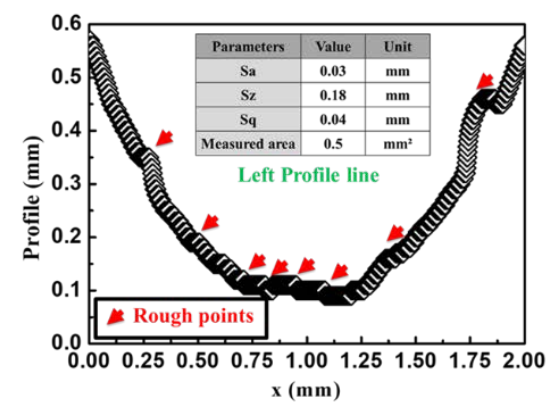

Figure 2. TENG output amplifier (TENG Amp.). (a) Exterior and interior design of TENG Amp and real TENG Amp. (b) Principle of TENG Amp. during clockwise rotation of vibration. (c) Principle of TENG Amp. during counter clockwise rotation of vibration. (d) Real mass and bar. (e) Rough contact surface in a hole shape, which functions similar to a lightning rod. (f) Roughness profiles of contact surface of right part. (g) Roughness profiles of contact surface of left part.

The basic resistance of the conductive sponge was measured to be $20 \mathrm{ohms}$. The specimen was completed by covering the exterior with silicone rubber, and a part of the silicone rubber penetrated into the inside pores. The TENG specimen was perfectly prepared when an electrode wire was plugged in. The specimen was $25 \mathrm{~mm}$ in radius and $9 \mathrm{~mm}$ in thickness (Figure 3c). 
(a)
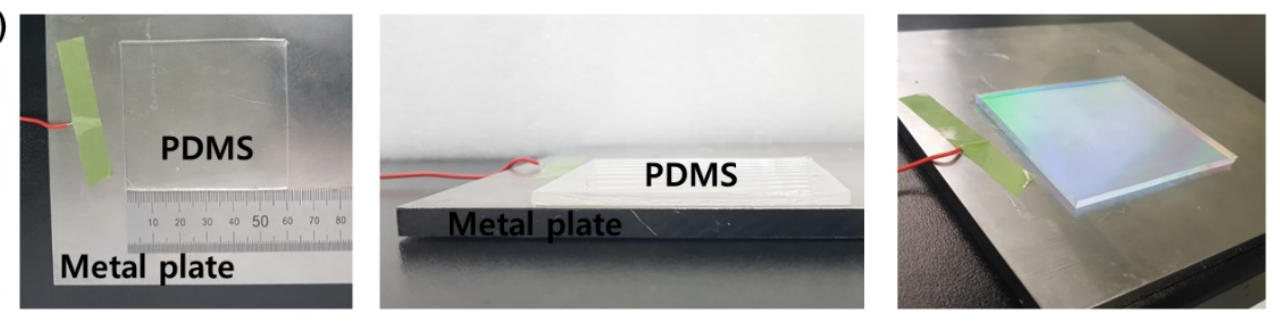

(b) Sugarmix
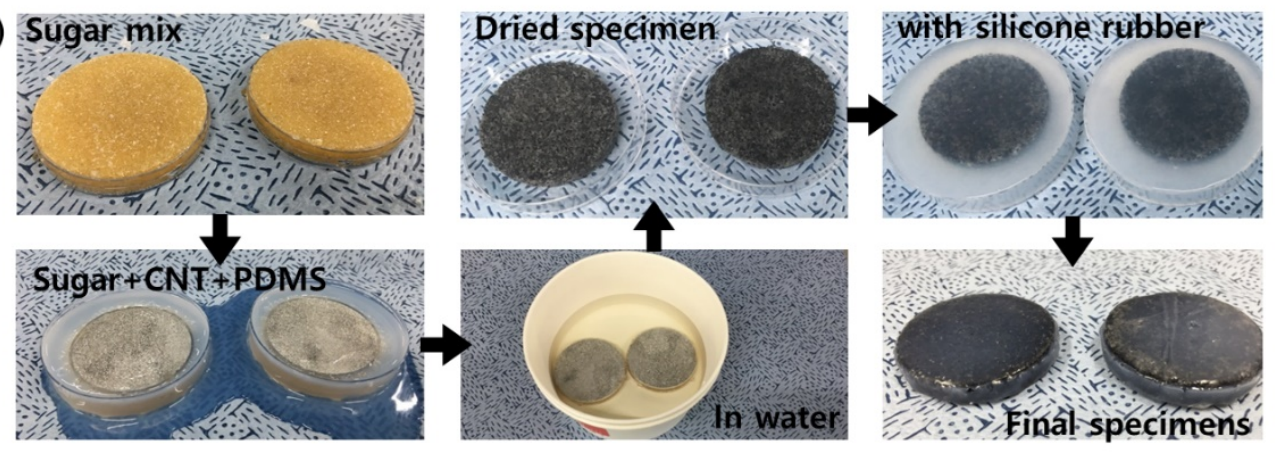

(c)
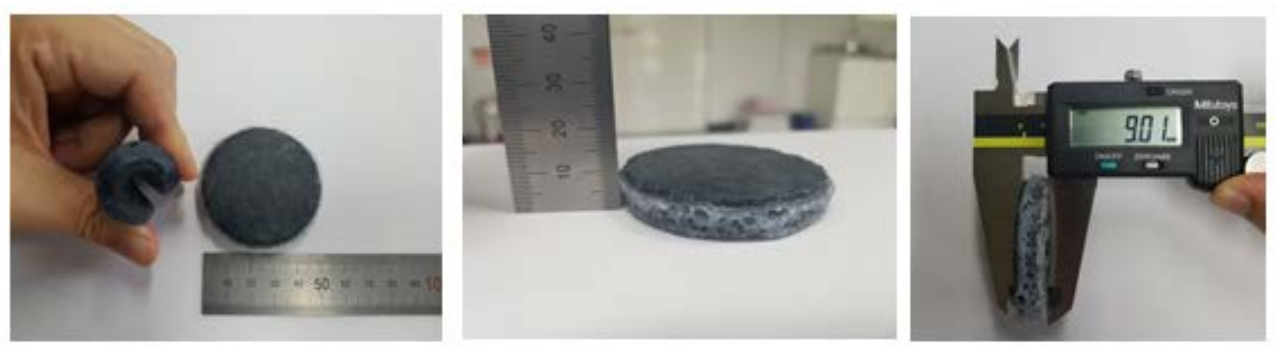

Figure 3. TENG test specimens: (a) PDMS-based TENG specimens; (b) fabrication process of conductive sponge TENG specimens; (c) photos of highly deformable conductive sponge TENG specimens.

\section{Results and Discussion}

In our test, the impact plate of a pushing tester (SPG-01, ATsolution) shown in Figure 4 was used as the charged counterpart causing triboelectrification. The impact plate was made of aluminum, and the test was performed at a frequency of $7 \mathrm{~Hz}$ and force of $0.7 \mathrm{~N}$. The pushing tester was driven by an AC speed control motor, repeatedly pushing the plate vertically. The TENG specimen generated triboelectrification by applying repeated pressure at $4-\mathrm{cm}$ intervals due to the pushing tester, and the contact/separation triboelectrification mode was adopted. As for the testing conditions, the temperature and humidity were $27^{\circ} \mathrm{C}$ and $50 \% \mathrm{RH}$, respectively.

The output of a TENG specimen was measured using the following devices: an oscilloscope (TBS2072, Tektronix Co., Beaverton, OR, USA), a high-voltage passive probe (P5100A, Tektronix Co.), and a low noise current amplifier (DLPCA-200, Femto Co. in Berlin, Germany). The output measurements of TENG specimens were compared with and without the output amplifier.

Figure 5a compares the output measurements of the simple PDMS-based TENG specimen with and without the TENG amplifier. Figure $5 \mathrm{~b}$ shows the output measurements depending on the resistance load. When the output amplifier was applied, both voltage and current were amplified, and the maximum output occurred at 10 mohm. 


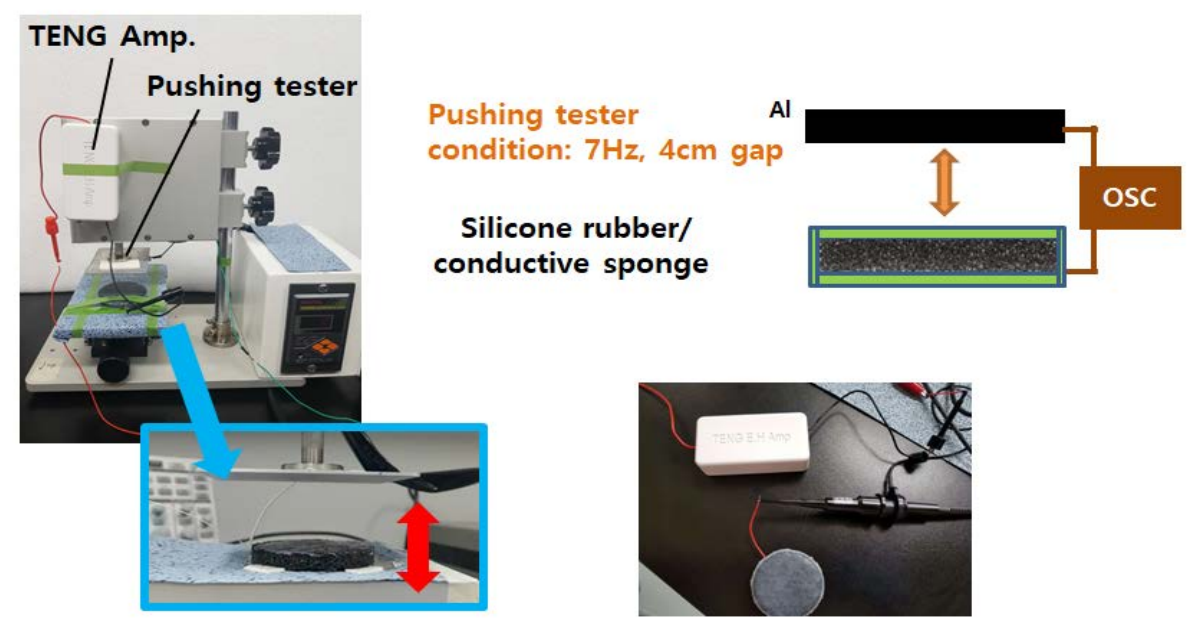

Figure 4. Measurement of output of the TENG specimen using the pushing tester.

Figure $5 c$,d shows the output measurements of the conductive CNT sponge TENG specimen. The porous specimen was pushed deeper at the same pressure. As the internal surface area of the porous specimen was larger than that of the PDMS-based TENG specimen, the basic output was observed to be similar in a smaller area. When the output amplifier was applied, the amplification was larger than in the PDMS specimen. This was because the TENG specimen with a higher charge density per unit area generated larger micro plasma inside the TENG amplifier.

The graphs in Figure $6 \mathrm{a}, \mathrm{b}$ enlarge a single interval of the conductive sponge specimen output. When the TENG output amplifier was used, the peak value of the output certainly increased. However, checking whether the effective power (that is, V rms and I rms) increased was still necessary. Table 1 thus presents the calculations of rms values. It turned out that rms values indicating that the effective power increased by over $20 \%$. Accordingly, the proposed TENG output amplifier could effectively increase the actual electric output.

Table 1. Comparison of rms values of TENG output performance with and without the amplifier.

\begin{tabular}{cccc}
\hline Output & $\begin{array}{c}\text { Voltage } \\
\text { (rms, V) }\end{array}$ & $\begin{array}{c}\text { Current } \\
\text { (rms, A) }\end{array}$ & $\begin{array}{c}\text { Power } \\
\text { (rms, W) }\end{array}$ \\
\hline w/o TENG Amp. & 0.7794 & $6.753 \times 10^{-5}$ & $5.26 \times 10^{-5}$ \\
w/ TENG Amp. & 0.9654 & $8.41 \times 10^{-5}$ & $8.12 \times 10^{-5}$ \\
\hline
\end{tabular}

The above increase in the output of TENG is attributable to the increase in the number of flowing electrons that was, in turn, caused by micro plasma continuously occurring in a TENG output amplifier during an external vibration. In this regard, the voltage increased slightly, while the current increased considerably. As the peak value of the increased current was too high and occurred only for a short moment of $1 \mathrm{~ms}$ and below, the graphs of the current measured with an oscilloscope could not capture all current values. Those currents that did not appear in the graphs were peak currents occurring at an interval shorter than the sampling rate of the oscilloscope. As this study focused on the overall increase of effective power, such an instantaneous peak current occurring for $1 \mathrm{~ms}$ and below was excluded.

As the TENG output amplifier utilized the occurrence of micro plasma, its output could be stabilized only by constant micro plasma. Accordingly, if the original TENG has a low output, the threshold voltage for generating micro plasma cannot be satisfied, and the amplifier is therefore hardly applicable. However, if the probe structure for generating micro plasma is moved by vibration, a very short distance can be obtained due to vibration, similar to what was observed in this study. Thus, even if the threshold voltage is very low, 
the output of most TENGs can be amplified. The conducive sponge TENG specimen in this study had a low output $(<50 \mathrm{~V})$ that could be amplified due to the occurrence of plasma.

(a)

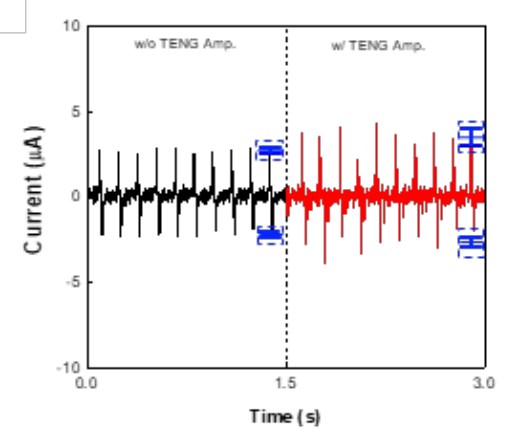

(b)

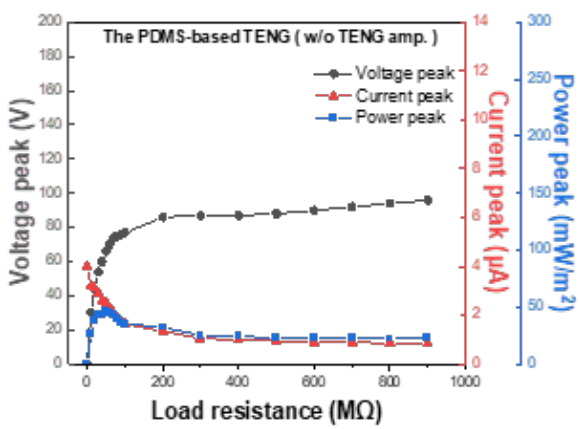

(c)

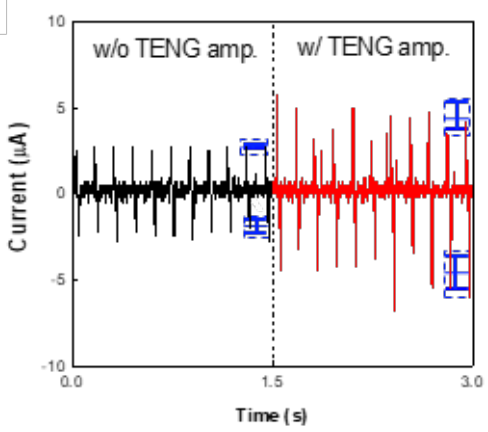

(d)

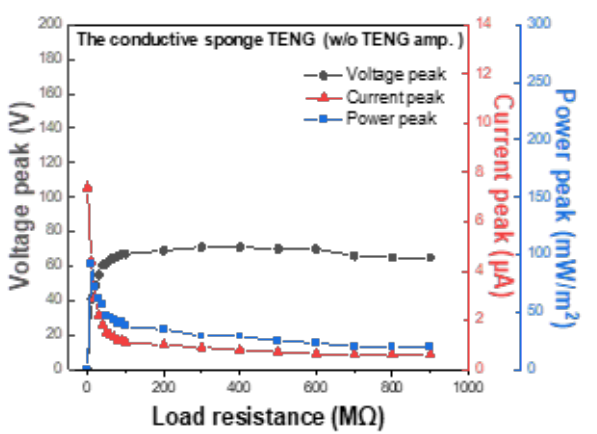

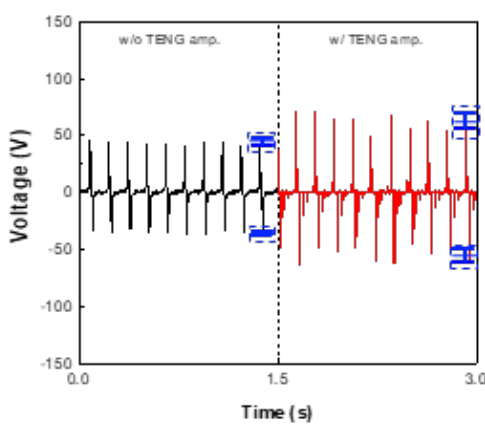
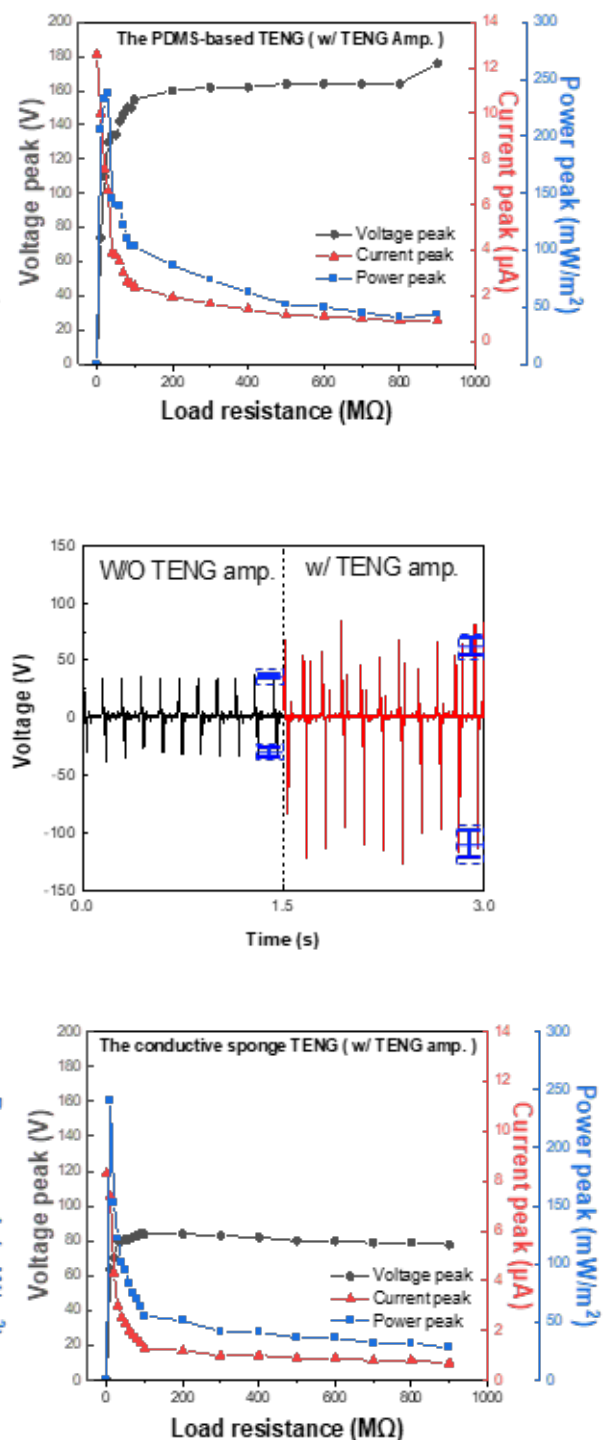

Figure 5. Comparison of outputs with and without the amplifier in a TENG specimen: (a) graphs of the current and voltage outputs of the PDMS-based TENG specimen; (b) graphs of the voltage peak, current peak, and power peak of the PDMS-based TENG specimen according to load resistance; (c) graphs of the current and voltage outputs of the conductive sponge TENG specimen; (d) graphs of the voltage peak, current peak, and power peak of the conductive sponge TENG specimen depending on load resistance. 


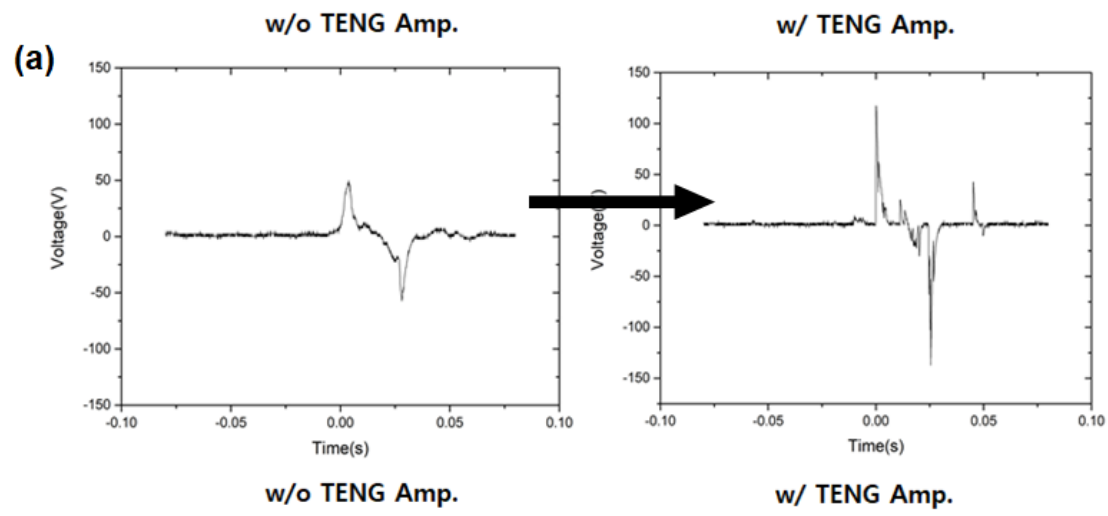

(b)

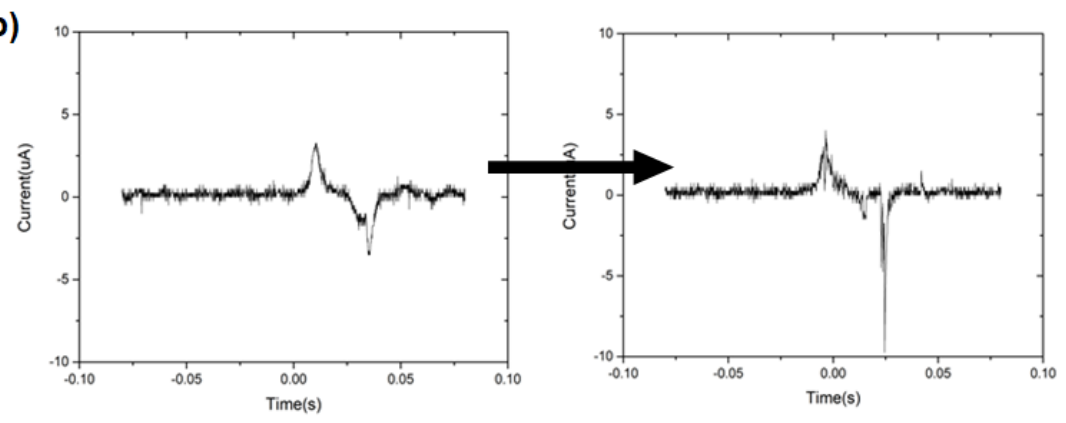

(c)

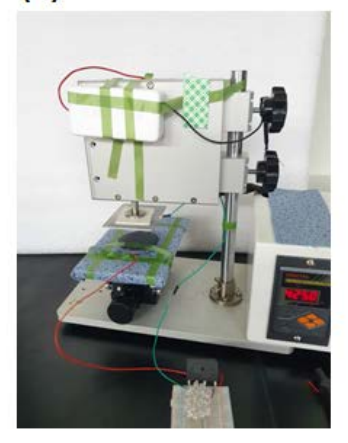

(d)

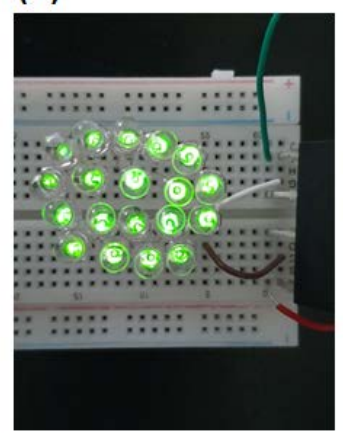

(e)

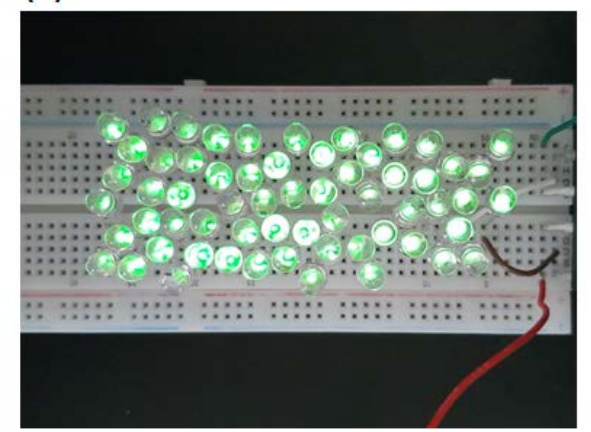

Figure 6. Comparison of the output performance of the conductive sponge TENG specimen with and without the amplifier: (a) comparison of voltage outputs for a single interval; (b) comparison of current outputs for a single interval; (c-e) comparison of the number of LEDs with and without the amplifier being connected to the conductive TENG specimen.

Multiple LEDs were used to visualize the effect of the TENG output amplifier. A rectifier was applied to the output terminal, which was connected to LEDs. The LEDs were connected in series. A pushing tester generated kinetic energies by applying the same force. As illustrated in Figure 6c-e, the conductive sponge TENG specimen without the amplifier could drive approximately 20 LEDs, while the one with the amplifier could drive approximately 60 LEDs.

\section{Conclusions}

In summary, this study tested a simple output amplifier applicable to most types of TENGs. As the amplifier can operate under an external vibration, and most TENGs generate electricity under vibrational conditions, the amplifier is effective in improving the output of almost all TENGs. The principle of an electron gun was utilized to accelerate the electron flow. In this manner, the power output could be instantaneously increased. When the gap distance changed by means of a vibrator, micro plasma occurred, which accelerated the electron flow. The vibrator can operate both in a vacuum and in an atmosphere. The TENG output amplifier could increase both voltage and current by 2-3 times. If this result 
is converted into area values, which are the values of effective power, it means that the overall output can be improved by roughly $20 \%$. Finally, it is expected that the size of the proposed TENG output amplifier will be reduced by applying a MEMS process.

Author Contributions: Conceptualization, methodology, data curation, writing-original draft, writing review and editing, J.P.; supervision, resources, writing—review and editing, H.C.; supervision, writing - review and editing, funding acquisition, Y.-S.L. All authors have read and agreed to the published version of the manuscript.

Funding: This work was supported by the Education and Research promotion program of KOREATECH in 2020 and the National Research Foundation of Korea (NRF) grant funded by the Korea government (MSIT) [2019R1C1C1010730, 2018R1D1A1B07040446, and NRF-2021R1G1A1012642].

Conflicts of Interest: The authors declare no conflict of interest.

\section{References}

1. Wang, Z.L.; Chen, J.; Lin, L. Progress in triboelectric nanogenerators as a new energy technology and self-powered sensors. Energy Environ. Sci. 2015, 8, 2250-2282. [CrossRef]

2. Bhatia, D.; Kim, W.; Lee, S.; Kim, S.W.; Choi, D. Tandem triboelectric nanogenerators for optimally scavenging mechanical energy with broadband vibration frequencies. Nano Energy 2017, 33, 515-521. [CrossRef]

3. Fan, F.-R.; Tian, Z.-Q.; Wang, Z.L. Flexible triboelectric generator. Nano Energy 2012, 1, 328-334. [CrossRef]

4. Zhu, G.; Lin, Z.-H.; Jing, Q.; Bai, P.; Pan, C.; Yang, Y.; Zhou, Y.; Wang, Z.L. Toward Large-Scale Energy Harvesting by a Nanoparticle-Enhanced Triboelectric Nanogenerator. Nano Lett. 2013, 13, 847-853. [CrossRef]

5. Tang, W.; Jiang, T.; Fan, F.R.; Yu, A.F.; Zhang, C.; Cao, X.; Wang, Z.L. Liquid-Metal electrode for High-Performance Triboelectric nanogenerator at an Instantaneous Energy Conversion Efficiency of 70.6\%. Adv. Funct. Mater. 2015, 25, 3718-3725. [CrossRef]

6. Fan, F.-R.; Lin, L.; Zhu, G.; Wu, W.; Zhang, R.; Wang, Z.L. Transparent Triboelectric Nanogenerators and Self-powered Pressure Sensors Based on Micropatterned Plastic Films. Nano Lett. 2012, 12, 3109-3114. [CrossRef] [PubMed]

7. Lee, K.Y.; Chun, J.; Lee, J.-H.; Kim, K.N.; Kang, N.-R.; Kim, J.-Y.; Kim, M.H.; Shin, K.-S.; Gupta, M.K.; Baik, J.M.; et al. Hydrophobic Sponge Structure-Based Triboelectric Nanogenerator. Adv. Mater. 2014, 26, 5037-5042. [CrossRef]

8. Xia, X.; Chen, J.; Liu, G.; Javed, M.S.; Wang, X.; Hu, C. Aligning graphene sheets in PDMS for improving output performance of triboelectric nanogenerator. Carbon 2017, 111, 569-576. [CrossRef]

9. Wang, S.; Xie, Y.; Niu, S.; Lin, L.; Liu, C.; Zhou, Y.S.; Wang, Z.L. Maximum Surface Charge Density for Triboelectric Nanogenerators Achieved by Ionized-Air Injection: Methodology and Theoretical Understanding. Adv. Mater. 2014, 26, 6720-6728. [CrossRef] [PubMed]

10. Chun, J.; Ye, B.U.; Lee, J.W.; Choi, D.; Kang, J.-Y.; Kim, S.-W.; Wang, Z.L.; Baik, J.M. Boosted output performance of triboelectric nanogenerator via electric double layer effect. Nat. Commun. 2016, 7, 12985. [CrossRef] [PubMed]

11. Chen, J.; Guo, H.; He, Z.; Liu, G.; Xi, Y.; Shi, H.; Hu, C. Enhancing Performance of Triboelectric Nanogenerator by Filling High Dielectric Nanoparticles into Sponge PDMS film. ACS Appl. Mater. Interface 2016, 8, 736-744. [CrossRef]

12. Xu, L.; Bu, T.Z.; Yang, X.D.; Zhang, C.; Wang, Z.L. Ultrahigh charge density realized by charge pumping at ambient conditions for triboelectric nanogenerators. Nano Energy 2018, 49, 625-633. [CrossRef]

13. Zhao, P.; Bhattacharya, G.; Fishlock, S.J.; Guy, J.G.M.; Kumar, A.; Tsonos, C.; Yu, Z.; Raj, S.; McLaughlin, J.A.; Luo, J.; et al. Replacing the metal electrodes in triboelectric nanogenerators: High-performance laser-induced graphene electrodes. Nano Energy 2020, 75, 104958. [CrossRef]

14. Mao, Y.; Zhao, P.; McConohy, G.; Yang, H.; Tong, Y.; Wang, X. Sponge-Like Piezoelectric Polymer Films for Scalable and Integratable Nanogenerators and Self-Powered Electronic Systems. Adv. Energy Mater. 2014, 4, 1301624. [CrossRef]

15. He, X.; Mu, X.; Wen, Q.; Wen, Z.; Yang, J.; Hu, C.; Shi, H. Flexible and transparent triboelectric nanogenerator based on high performance well-ordered porous PDMS dielectric film. Nano Res. 2016, 9, 3714-3724. [CrossRef]

16. Chun, J.; Kim, J.W.; Jung, W.-S.; Kang, C.-Y.; Kim, S.-W.; Wang, Z.L.; Baik, J.M. Mesoporous pores impregnated with Au nanoparticles as effective dielectrics for enhancing triboelectric nanogenerator performance in harsh environments. Energy Environ. Sci. 2015, 8, 3006-3012. [CrossRef]

17. Kim, Y.J.; Lee, J.; Park, S.; Park, C.; Park, C.; Choi, H.-J. Effect of the relative permittivity of oxides on the performance of triboelectric nanogenerators. RSC Adv. 2017, 7, 49368-49373. [CrossRef]

18. Hwang, H.J.; Lee, Y.; Lee, C.; Nam, Y.; Park, J.; Choi, D.; Kim, D. Mesoporous Highly-Deformable Composite Polymer for a Gapless Triboelectric Nanogenerator via a One-Step Metal Oxidation Process. Micromachines 2018, 9, 656. [CrossRef] [PubMed]

19. Lee, J.-H.; Yoon, H.-J.; Kim, T.Y.; Gupta, M.K.; Lee, J.H.; Seung, W.; Ryu, H.; Kim, S.-W. Micropatterned P(VDF-TrFE) Film-Based Piezoelectric Nanogenerators for Highly Sensitive Self-Powered Pressure Sensors. Adv. Funct. Mater. 2015, 25, 3203-3209. [CrossRef] 
20. Lee, J.; Hinchet, R.; Kim, S.K.; Kim, S.; Kim, S.-W. Shape memory polymer-based self-healing triboelectric nanogenerator. Energy Environ. Sci. 2015, 8, 3605. [CrossRef]

21. Kim, J.; Cho, H.; Han, M.; Jung, Y.; Kwak, S.S.; Yoon, H.-J.; Park, B.; Kim, H.; Kim, H.; Park, J.; et al. Ultrahigh Power Output from Triboelectric Nanogenerator Based on Serrated Electrode via Spark Discharge. Adv. Energy Mater. 2020, 10, 2002312. [CrossRef] 